\title{
In Situ Detection of Neurotransmitters from Stem Cell- derived Neural Interface at the Single-Cell Level via Graphene-Hybrid SERS Nanobiosensing
}

Jin-Ha Choi ${ }^{\dagger,}$, Tae-Hyung Kim ${ }^{\dagger,}$, Waleed Ahmed El-said ${ }^{\ddagger}, \perp$, Jin-Ho Lee $^{\dagger,}$, Letao Yang $^{\dagger}$, Brian Conley ${ }^{\dagger}$, JeongWoo Choi ${ }^{* \neq}$ and Ki-Bum Lee ${ }^{*,+}$

${ }^{\dagger}$ Department of Chemistry and Chemical Biology, Rutgers, The State University of New Jersey, 123 Bevier Road, Piscataway, NJ 08854, USA

${ }^{\ddagger}$ Department of Chemical \& Biomolecular Engineering, Sogang University, 35 Baekbeom-ro, Mapo-gu, Seoul 04107, Republic of Korea

§Present Address: School of Integrative Engineering, Chung-Ang University, 84 Heukseok-ro, Dongjak-gu, Seoul 06974, Republic of Korea

${ }^{\perp}$ Department of Chemistry, Faculty of Science, Assiut University, Assiut 71516, Egypt

*Corresponding authors: jwchoi@sogang.ac.kr, kblee@rutgers.edu

*Website: http://kblee.rutgers.edu/

KEYWORDS: Surface-enhanced Raman scattering, Detections of Neurotransmitters, Single-cell analysis, Graphenehybrid SERS nanobiosensing. 


\section{EXPERIMENTAL SECTION}

Materials and cells: ITO electrodes were purchased from U.I.D., South Korea. Gold electrode, chromium (adhesion layer), and gold were deposited on the cover glass with a thickness of $2 \mathrm{~nm}$ and $50 \mathrm{~nm}$ using E-beam evaporator, respectively. All chemicals used in this study including gold (III) chloride hydrate, ammonium sulfate, potassium citrate tribasic monohydrate, dopamine, L-Dopa, 3,4-Dihydroxy-L-phenylalanine and Formalin solution (neutral buffered, 10\%) were purchased from Sigma-Aldrich, USA. All the materials used for the photolithographic process including photoresist (AZ2020), solvent (AZ EBR Solvent), developer (AZ MIF3000) and stripper (AZ 400T) were obtained from AZ Electronic Materials, USA. SYLGARD 184 silicone elastomer kit (Polydimethylsiloxane, PDMS) was purchased from Dow Corning, USA. For cell culture, Dulbecco's modified eagle's medium (DMEM), 0.05\% trypsin, and Penicillin/Streptomycin (PS) were purchased from Life Technologies, USA. Phosphate buffered saline (PBS) was obtained from Sigma-Aldrich, USA. Heat-inactivated fetal bovine serum (FBS) was purchased from Gibco, USA.

PC-12 $(\mathrm{p}=15)$ cells, which were derived from a pheochromocytoma of the rat adrenal medulla, were purchased from ATCC, USA and were utilized as a model dopaminergic cell line in this study. Rat neural PC-12 cells and NE-4C, a non-dopaminergic undifferentiated neural stem cell from mouse, were cultured in DMEM supplemented with $10 \%$ heat-inactivated FBS and 1\% PS. The cells were maintained under standard cell culture conditions at $37^{\circ} \mathrm{C}$ in an atmosphere of $5 \% \mathrm{CO}_{2}$. The medium was changed every three days. The number of cells was determined by the trypan blue assay with a hemocytometer.

ReNcell VM Cells (ReN cells) were used to achieve dopaminergic neurons, as previously described. Specifically, on day 1, 1.5 million ReN cells were placed in a $10 \mathrm{~cm}$ diameter tissue culture plastic (TCP) and treated with the medium (mixture of neurobasal medium and DMEM/F 12 medium with ratio of 1:1) containing $20 \mathrm{ng} / \mathrm{ml}$ of epidermal growth factor (EGF) and $20 \mathrm{ng} / \mathrm{ml}$ of basic fibroblast growth factor (bFGF) to generate neurospheres. After 7 days of incubation in normal culture condition $\left(37^{\circ} \mathrm{C}, 5 \% \mathrm{CO}_{2}\right)$, neurospheres were spun down at $1.0 \mathrm{k} \mathrm{rev} / \mathrm{min}$ for $60 \mathrm{sec}$ and transferred to a nano-SERS array, followed by further incubation with the medium supplemented with $20 \mathrm{ng} / \mathrm{ml}$ of EGF and bFGF. On day 10,2 $\mathrm{ng} / \mathrm{ml}$ of glial cell-derived neurotrophic factor (GDNF) and $1 \mu \mathrm{M}$ of dibutyl-cyclic adenosine monophosphate (cAMP) were also introduced to cell culture media on day 10 for dopaminergic differentiation without EGF and bFGF. On day 17, the differentiation process was completed and after that, the cell culture medium was replaced every 3 days without the addition of growth factors and small molecules.

Development of the GO modified homogeneous gold nanoarray: The hybrid nano-SERS array fabrication is described in our previous report ${ }^{[8]}$. Briefly, ITO substrates (UID South Korea) were sonicated with $1 \%$ Triton X100 , deionized water (DIW), and 95\% Ethanol for 20 min each and dried with $\mathrm{N}_{2}$ gas. Next, hexamethyldisilazane (HMDS) was spin-coated (Laurell Technologies USA) onto cleaned ITO and negative photoresist AZ 2020 (AZ 
Electronic Materials, USA) at a dilution of 1:0.8 AZ EBR solvent was spin-coated onto ITO substrates. Afterward, substrates were pre-baked for $60 \mathrm{~s}$ at $125^{\circ} \mathrm{C}$ and exposed to $325 \mathrm{~nm}$ light at $0.78 \mathrm{~mW}$ (He-Cd Laser, KIMMON KOHA Laser Systems, Japan) with a Lloyd's Mirror Interferometer (Thor Labs). Following exposure, substrates were baked for $60 \mathrm{~s}$ at $125^{\circ} \mathrm{C}$. The excess photoresist was wet etched with AZ MIF 3000 Developer solution and washed with DIW. Substrates were dried with $\mathrm{N}_{2}$ gas, subjected to oxygen plasma $\left(140 \mathrm{~W}, 50 \mathrm{sccm} \mathrm{O}_{2}\right)$ and baked for $60 \mathrm{~s}$ at $135{ }^{\circ} \mathrm{C}$. To electrochemically deposit gold, circular plastic chambers approximately $0.5 \mathrm{~cm}$ in height and $0.5 \mathrm{~cm}$ in diameter were annealed to the PR nanopattern surface using PDMS (10:1). Next, a gold solution comprised of $5 \mathrm{mM} \mathrm{HAuCl}_{4}, 0.5 \mathrm{mM}\left(\mathrm{NH}_{4}\right)_{2} \mathrm{SO}_{4}$, and $2 \mathrm{mM} \mathrm{KCl}$ were pipetted into the chamber, and DC amperometry (Epsilon Potentiostat, BASi, USA) was performed at a potential of $-1.2 \mathrm{~V}$ for $240 \mathrm{~s}$. The excess photoresist was then stripped using photoresist stripper $\mathrm{AZ} 400 \mathrm{~T}$ for $70 \mathrm{~min}$ at $65^{\circ} \mathrm{C}$ and washed with acetone, DIW, and ethanol. After making the gold nanoarray, it was subsequently cleaned by a $50 \mathrm{mM} \mathrm{KOH}$ solution with $25 \% \mathrm{H}_{2} \mathrm{O}_{2}$ for $15 \mathrm{~min}$. For the modification of the $\mathrm{GO}$ on the homogeneous gold nanoarray, $1 \mathrm{mM}$ cysteamine solution was covered on the whole substrate for 180 min and wash 3 times with DIW. After this, GO solution (Sigma-Aldrich, USA) is placed on the homogeneous gold nanoarray and incubated overnight at room temperature (RT).

Finite Difference Time Domain (FDTD) simulation: To study the enhancement of the electric field induced by our nanoarray, we performed FDTD simulation based on parameters used in the SERS experiments. Briefly, based on the AFM data and SEM data in Figure 2, we built the 3D model for individual nanostructures with teeth-like structure (symmetrically assembled from six parabola cone structures with heights of $250 \mathrm{~nm}$ ) and with the composition of gold $[\operatorname{Re}($ index $)=0.08$ and $\operatorname{Im}($ index $)=4.56$ at the wavelength of $780 \mathrm{~nm}]$. Then a four by four array was built from the teeth-like nanostructure on top of a silicon substrate $[\operatorname{Re}($ index $)=3.714$ and $\operatorname{Im}($ index $)=0.008$ at the wavelength of $780 \mathrm{~nm}]$. To match the laser wavelength used in the SERS measurements, light source (plane wave) with a wavelength of $780 \mathrm{~nm}$ and vertical to the substrate was placed on top of the plasmonic structures. To perform the FDTD simulation, we defined 4nm mesh size and used a perfectly matched layer (PML) boundary conditions with 12-layer numbers. The media surrounding the plasmonic nanoarrays is set to vacuum. The vertical and parallel monitors (base plane: supporting substrate) selectively monitor light with a wavelength of $780 \mathrm{~nm}$ to be consistent with the light source. For the gold nanoparticle (diameter $=250 \mathrm{~nm}) \mathrm{control}$, the mesh size, boundary condition and other simulation methods in FDTD, and the material settings are kept identical. The distributions of $\mathrm{E} / \mathrm{E}_{0}$ as a function of the positions $[(\mathrm{x}, \mathrm{y})$ and $(\mathrm{x}, \mathrm{z})]$ are the final result visualized by the heatmap image summarized in Figure $2 \mathrm{~g}$.

Detection of DA by using an aptamer-MG modified hybrid nano-SERS array: DA specific aptamer was synthesized by STPharm (Korea) with a sequence of 5 -GTC TCT GTG TGC GCC AGA GAA CAC TGG GGC AGA TAT GGG CCA GCA CAG AAT GAG GCC C-3`-Malachite Green (MG). The aptamer-MG complex (1 
$\mu \mathrm{M}$ ) was adsorbed on the GO surface overnight at $4{ }^{\circ} \mathrm{C}$. Next, it was washed 5 times using $0.01 \mathrm{M}$ PBS solution with $0.05 \%$ Tween 20 (Sigma-Aldrich, USA). For the DA reaction on the aptamer-modified hybrid nano-SERS array, various concentrations from $10^{-9}$ to $10^{-3} \mathrm{M}$ of DA solution were prepared and reacted for 60 min. After washing with PBS, Raman intensity was investigated based on the SERS technique using Raman NTEGRA Spectra (NT-MDT, Russia) equipped with an inverted optical microscope and a liquid nitrogen-cooled CCD detector. The maximum scan range $(\mathrm{x}, \mathrm{y}, \mathrm{z})$ was $100 \mathrm{~mm} \times 100 \mathrm{~mm} \times 6 \mathrm{~mm}$; while the movement limitation was $200 \mathrm{~nm}$ in the XY plane and $500 \mathrm{~nm}$ along the Z-axis. The resolution of the Raman spectrometer was $785 \mathrm{~nm}$ in the XYZ plane. Raman spectra were recorded using a NIR laser-emitting light at a wavelength of $785 \mathrm{~nm}$, with an irradiation laser power of $3 \mathrm{~mW}$ on the sample plane. Ten scans of $1 \mathrm{~s}$ from $300-1800 \mathrm{~cm}^{-1}$ were recorded, and the mean result was used. A blank spectrum was acquired before each step, which allowed the absorbance to be subsequently measured.

Detection of releasing DA from the single dopaminergic cell: The small plastic chamber was first attached to the surface of the hybrid nano-SERS array using PDMS with a ratio of 10:1 to culture PC-12 cells. Cells were seeded on the aptamer-modified hybrid nano-SERS array (approximately 50,000 cells/chip) and incubated 1 day for stable attachment on the surface. $100 \mu \mathrm{M}$ of L-Dopa was dissolved in media and was treated for 2 hours before the detection by Raman NTEGRA Spectra. The media was changed to PBS (0.01M, PH 7.4) containing $5 \mathrm{mM}$ $\mathrm{KCl}$ to trigger dopamine secretion from dopaminergic cells (PC-12). For the monitoring of neural differentiation, ReN cells were induced differentiation using differentiation media and measured by Raman mapping without $\mathrm{KCl}$ and L-Dopa treatment. Next, Raman intensity was determined by Raman NTEGRA Spectra with a liquid nitrogen-cooled CCD detector. The XY scan-range was varied, from $50 \mu \mathrm{m} \times 50 \mu \mathrm{m}$ to $100 \mu \mathrm{m} \times 100 \mu \mathrm{m}$; the resolution of the spectrometer was $785 \mathrm{~nm}$ in the XYZ plane; The laser spot size is around $1 \mu \mathrm{m}$ and the minimum step size is above $1 \mu \mathrm{m}$; the mapping points were $32 \times 32$. The total points in one mapping image were 1,024 and the exposure time of each point was 1s. Raman spectra were recorded using a NIR laser-emitting light at a wavelength of $785 \mathrm{~nm}$, with an irradiation laser power of $3 \mathrm{~mW}$ on the sample plane. The cell culture media was removed, and the cells were washed three times with PBS, before SERS measurements to eliminate the effect of the medium on SERS signals. Ten scans of $1 \mathrm{~s}$ exposure from 300-1800 $\mathrm{cm}^{-1}$ are recorded, and the mean result was used. A blank spectrum was acquired before each step, which allowed the absorbance to be subsequently measured. For the normalization of SERS signals, as well as remove background noises, particular software (Labspec5) was utilized with the same conditions. 

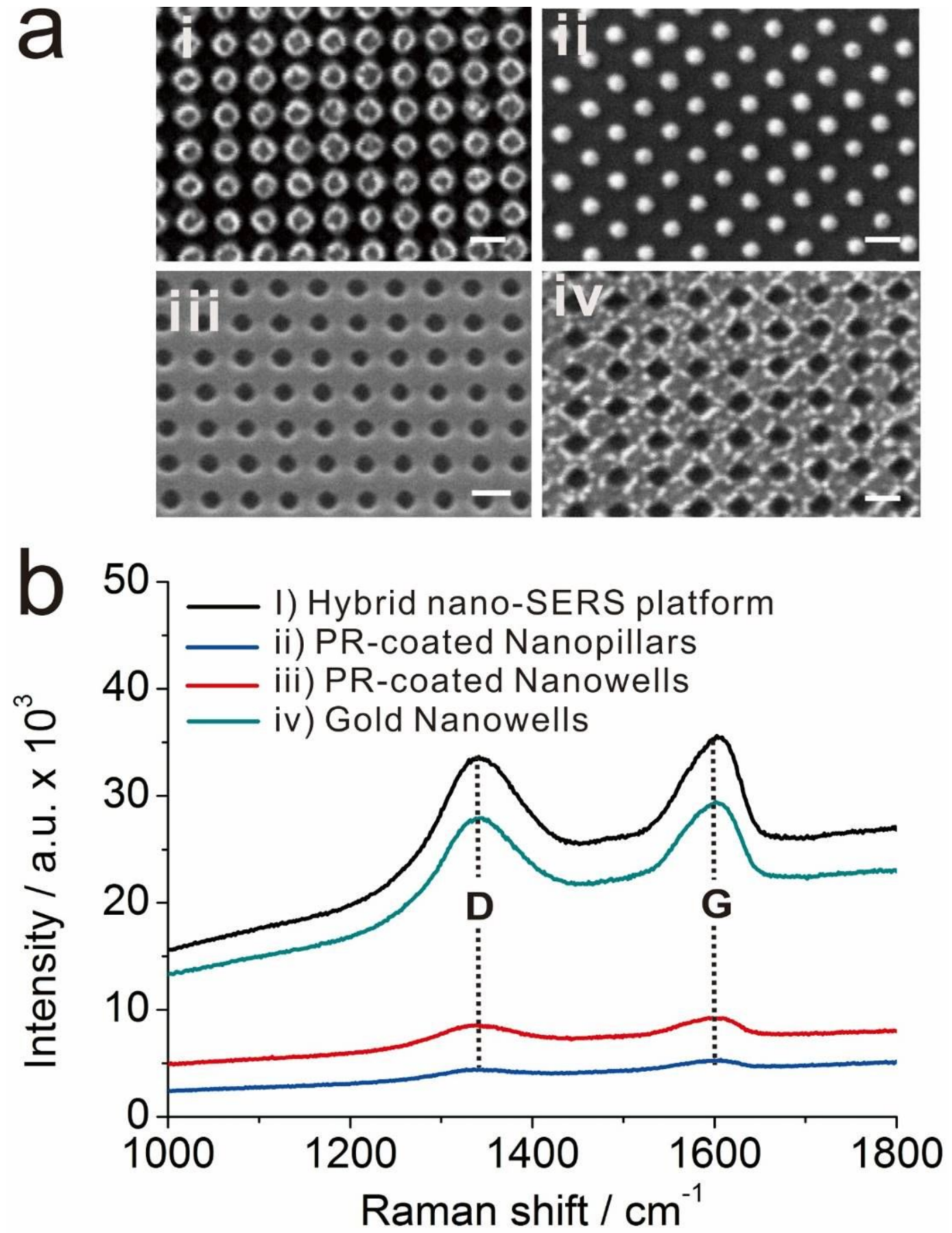

Figure S1. Raman spectra of the different shape of graphene oxide (GO) modified nanoarrays. (a) Scanning electron microscopic (SEM) images of i) graphene oxide (GO)-hybrid nano-SERS array, ii) homogeneous goldcoated photoresist nanopillar arrays, iii) homogeneous gold-coated photoresist nanowell arrays, and iv) homogeneous gold nanowell arrays. (b) Raman spectra GO on the four different gold structures. The hybrid nanoSERS array shows the highest $D\left(1350 \mathrm{~cm}^{-1}\right)$ and $\mathrm{G}\left(1600 \mathrm{~cm}^{-1}\right)$ peaks among all four different substrates. Scale Bar $=500 \mathrm{~nm}$ 


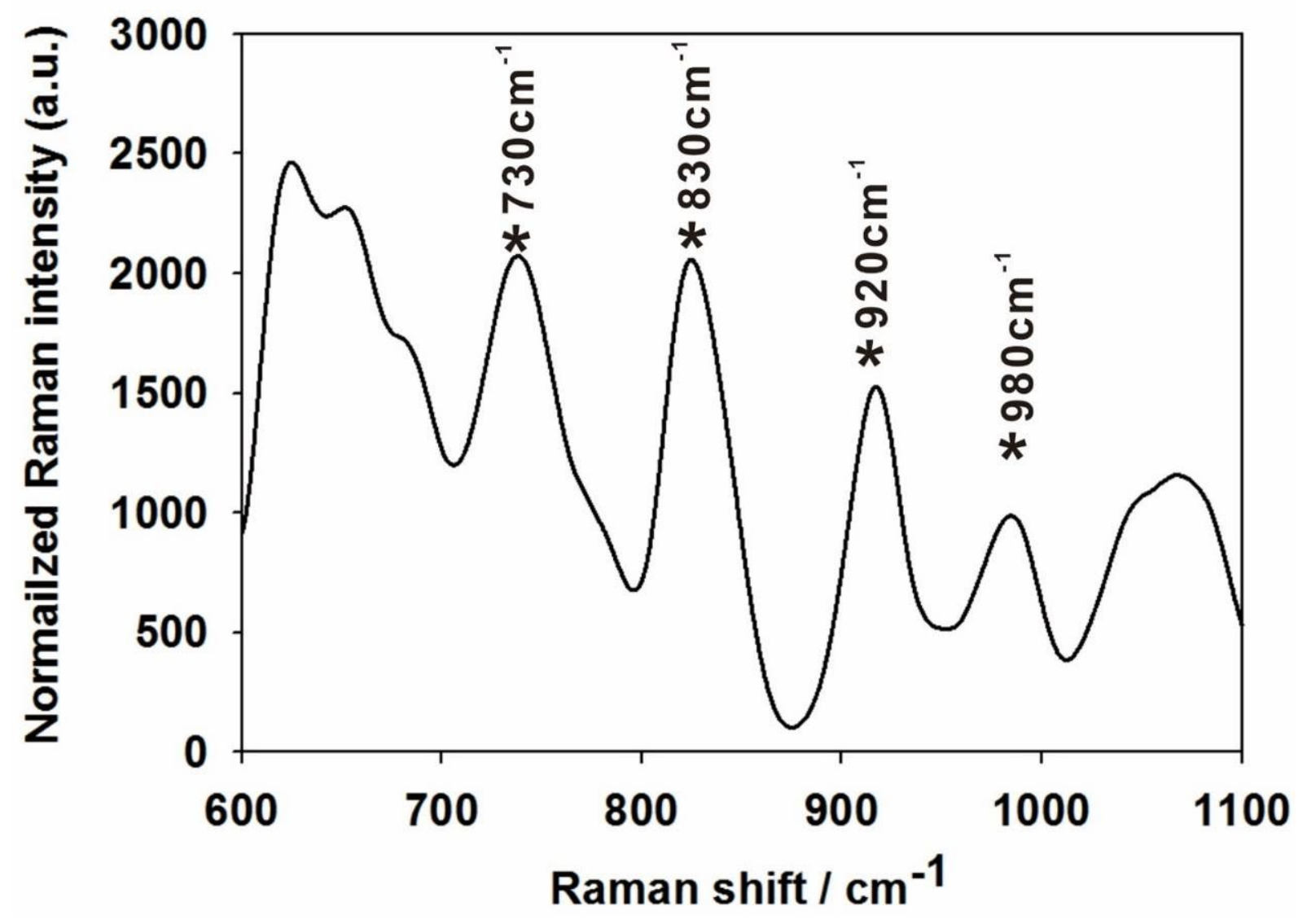

Figure S2. Raman spectra of the malachite green (MG)-modified dopamine (DA) aptamers. Raman spectra obtained from the MG-modified DA aptamers on the hybrid nano-SERS array. The Raman peaks used for both quantitative detection of the chemical dopamine in cell-free configuration and dopamine release from single neuronal cells were marked as the asterisk $(*)$. 


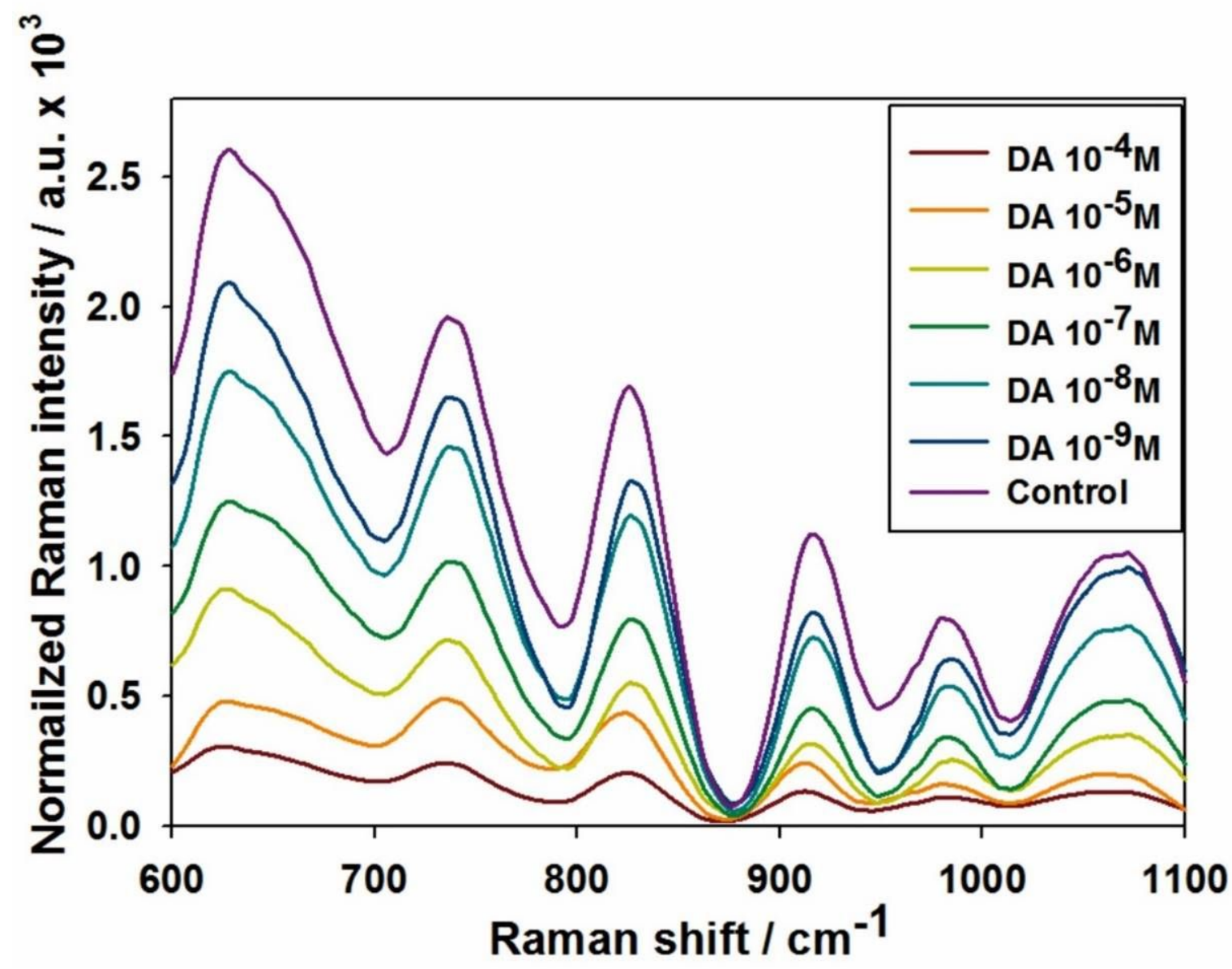

Figure S3. Raman spectra of MG-aptamer modified hybrid nano-SERS array after reaction of chemical DA in the cell-free configuration for the quantitative detection. Raman spectra used for the quantitative detection of the chemical dopamine in cell-free configuration corresponded with figure 4. Four different Raman peaks (730 $\mathrm{cm}^{-1}, 830 \mathrm{~cm}^{-1}, 920 \mathrm{~cm}^{-1}$, and $980 \mathrm{~cm}^{-1}$ ), which are Raman peaks of MG, not the DA, used for the detection of the chemical dopamine. 

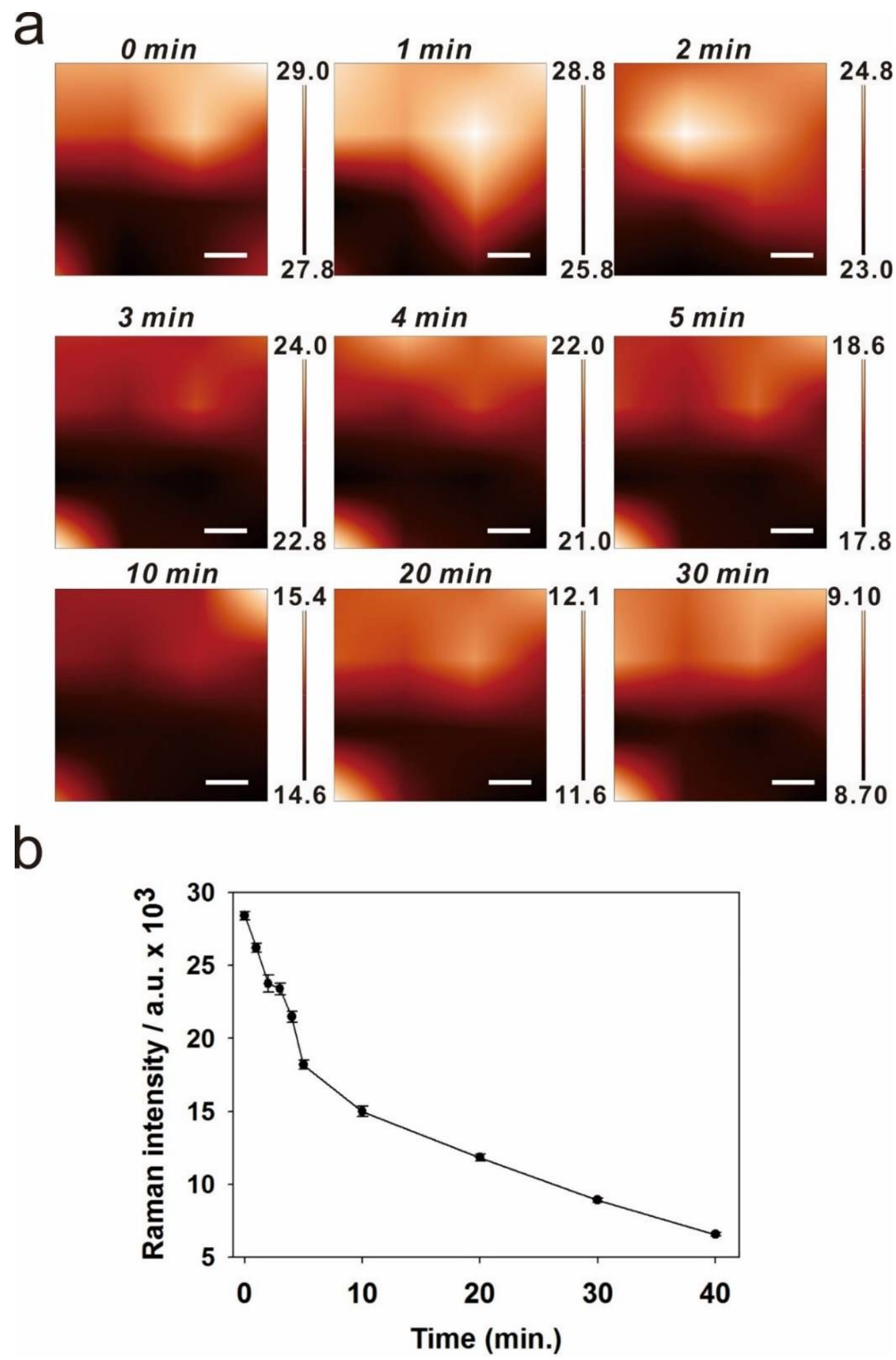

Figure S4. Time-dependent Raman mapping image of hybrid nano-SERS array with the addition of $10 \mu M$ of $\boldsymbol{D} \boldsymbol{A}$. (a) Raman mapping images at $830 \mathrm{~cm}^{-1}$ of MG-aptamer modified hybrid nano-SERS array with the addition of DA in a time-dependent manner. After 40 minutes of incubation, the Raman intensities were decreased owing to the mass loss of DA aptamers adsorbed on the surface of the hybrid nano-SERS array. The unit of the scale is $10^{3}$. Scale bars are $20 \mu \mathrm{m}$. (b) Raman intensities from Raman mapping images in (a). Data were expressed as mean \pm S.E.M. $(n=10)$. 
a
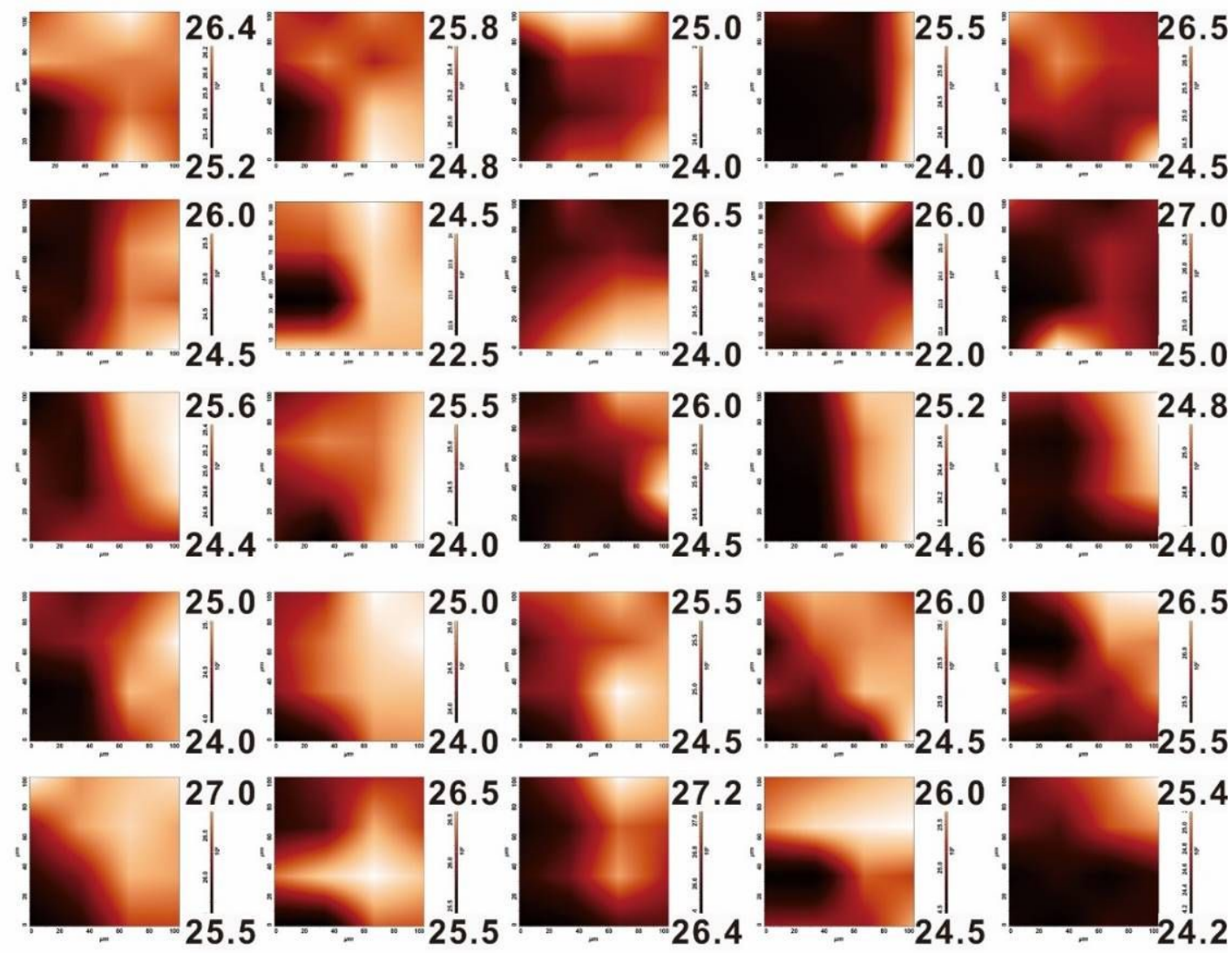

b

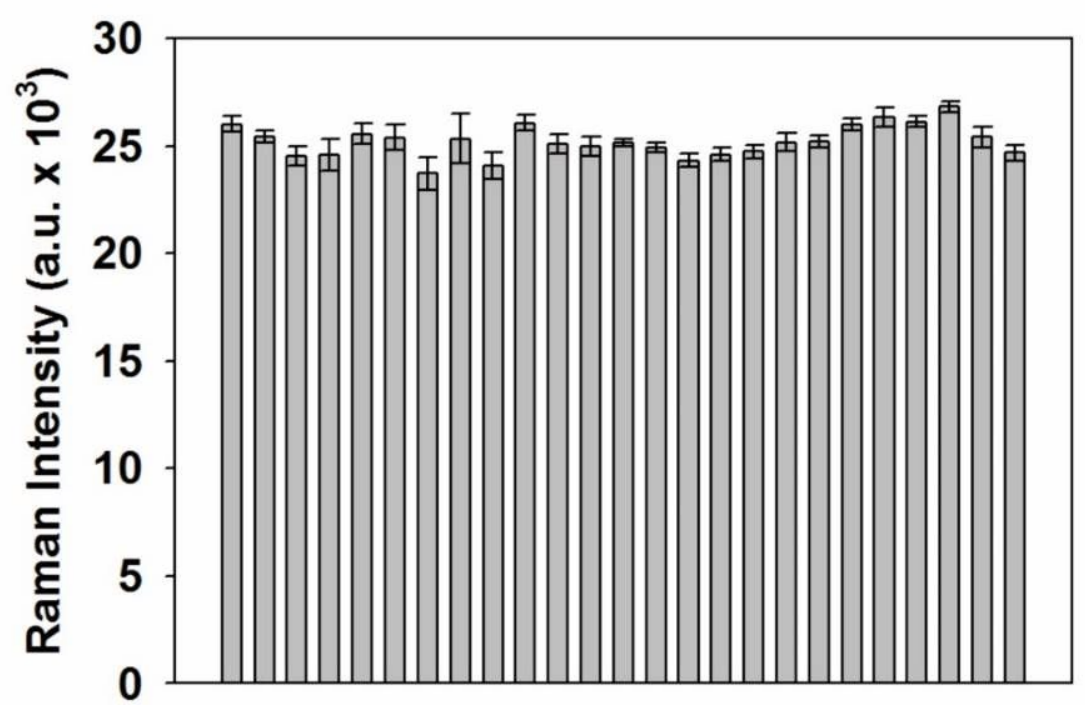

Figure S5. Homogeneity of the Raman signal on the hybrid nano-SERS array. (a) Raman mapping images sized $500 \times 500 \mu \mathrm{m}$, divided into 25 areas. Each of the image sizes was $100 \times 100 \mu \mathrm{m}$. The unit of the Raman intensity was the $10^{3}$ order. Twenty-five Raman mapping images were taken with the similar Raman intensities at $830 \mathrm{~cm}^{-1}$. Scale bars are $20 \mu \mathrm{m}$. (b) The Raman intensities of each 25 mapping images with error bars. 


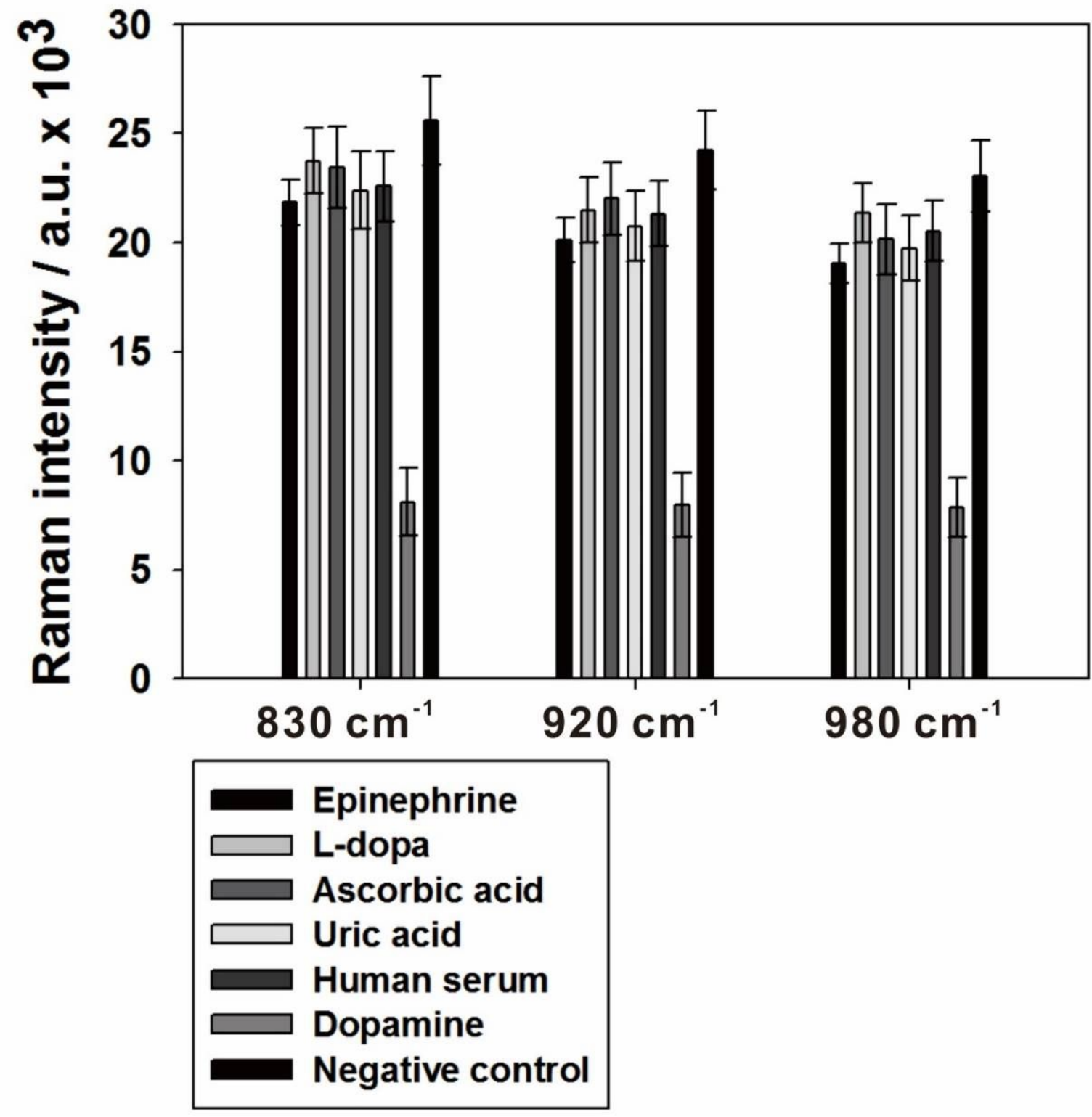

Figure S6. Raman intensities of the MG-specific peaks after applying other molecules. Raman intensities of MG-aptamer modified hybrid nano-SERS array with the addition of ten micromolar of epinephrine, L-dopa, ascorbic acid uric acid, which has a similar structure to DA, and human serum without DA and DA molecule. Three different Raman peaks ((a) $830 \mathrm{~cm}^{-1}$, (b) $920 \mathrm{~cm}^{-1}$, and (c) $980 \mathrm{~cm}^{-1}$, respectively) were obtained and averaged in the graph. 
a

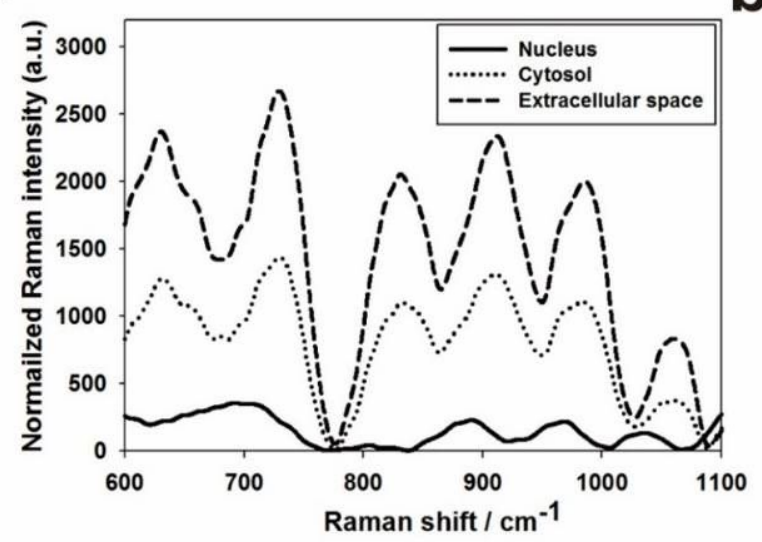

C

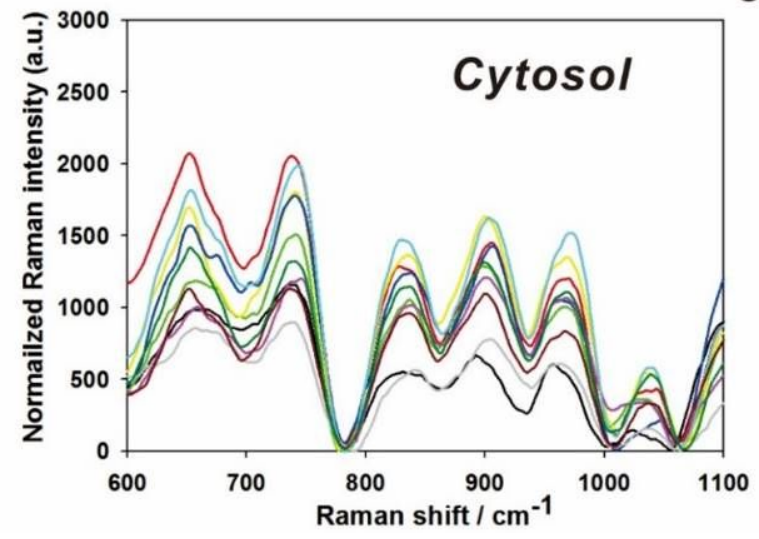

b

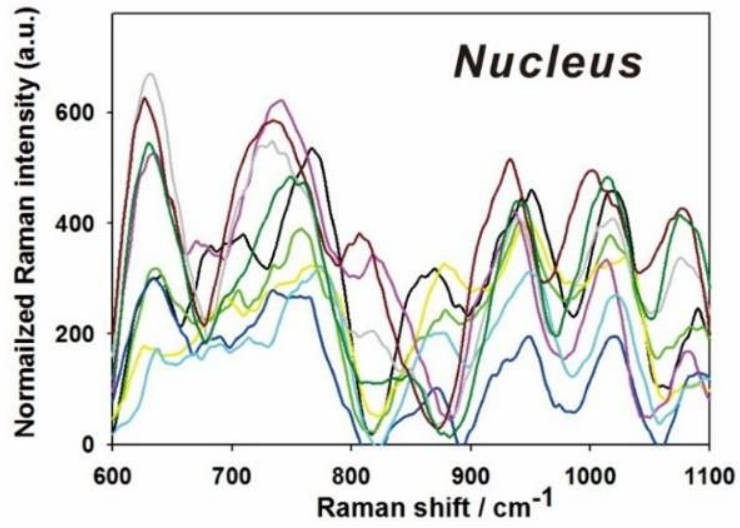

d

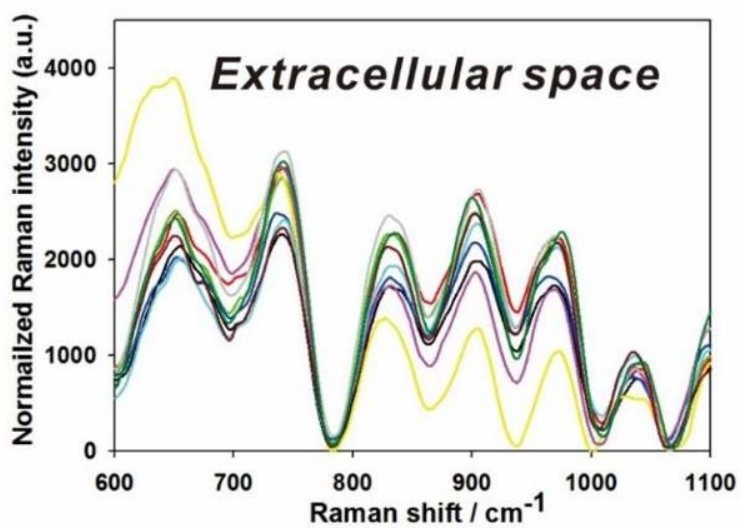

Figure S7. Raman spectra obtained from different areas of the PC-12 cell on the hybrid nano-SERS array. (a) Averaged Raman spectra of the PC-12 cell on the MG-aptamer modified hybrid nano-SERS array. 'Averaged' spectra indicate that the Raman signals were averaged from 10 different points of Raman signals that were achieved from the same areas (e.g., cell nucleus, cytosol, and around cells). (b d) Raman spectra from the different PC-12 areas, which are in the (b) nucleus, (c) cytosol, and (d) the extracellular space. 


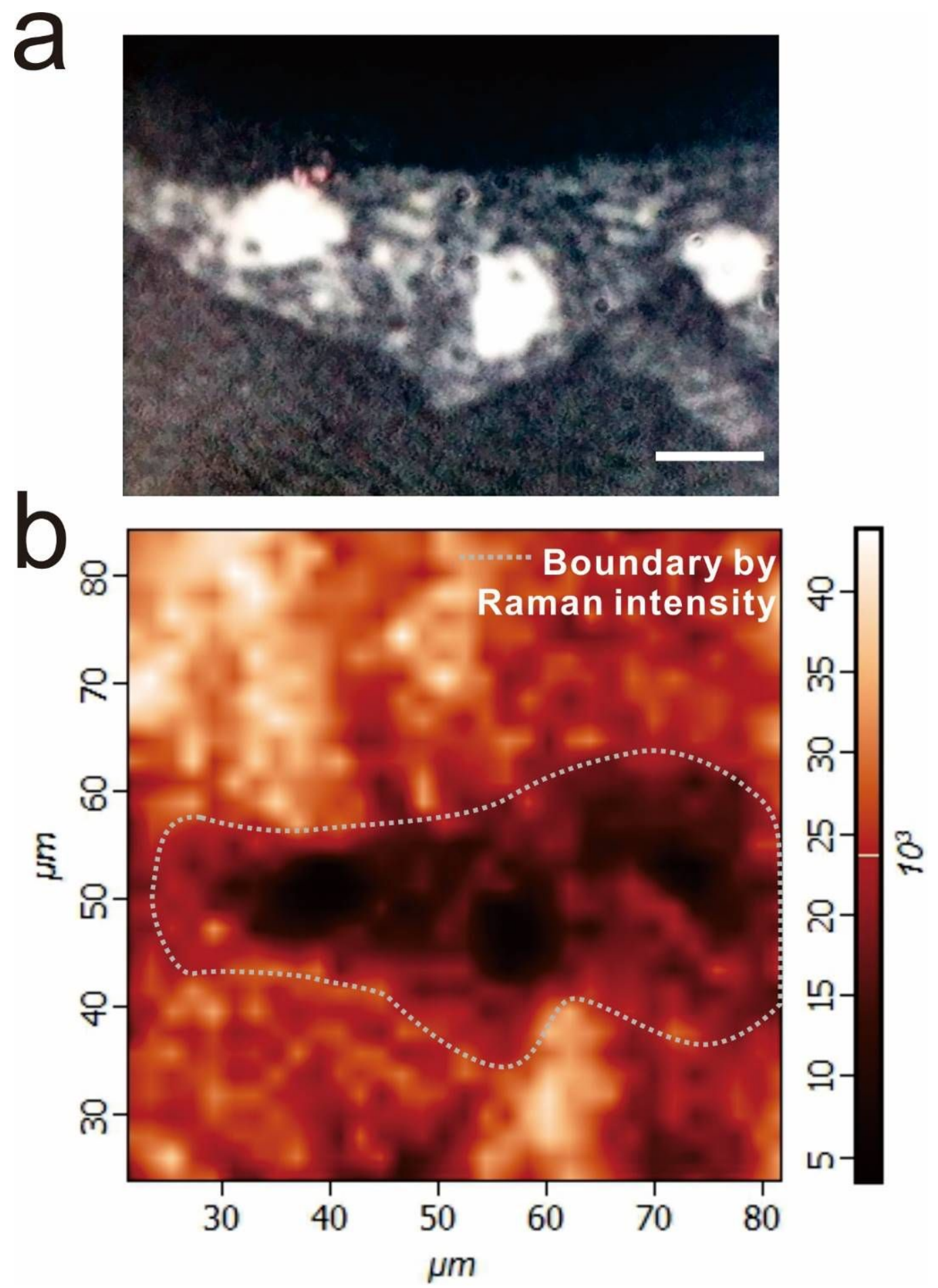

Figure S8. Images of multiple PC-12 cells on MG-aptamer modified hybrid nano-SERS array. (a) Phase contrast and (b) Raman mapping images at $830 \mathrm{~cm}^{-1}$ of multiple PC-12 cells on the aptamer-modified hybrid nano-SERS array. Inside of the dotted line indicates the release of DA from the dopaminergic cell body, based on the changes of Raman signals. The scale bar is $10 \mu \mathrm{m}$. 

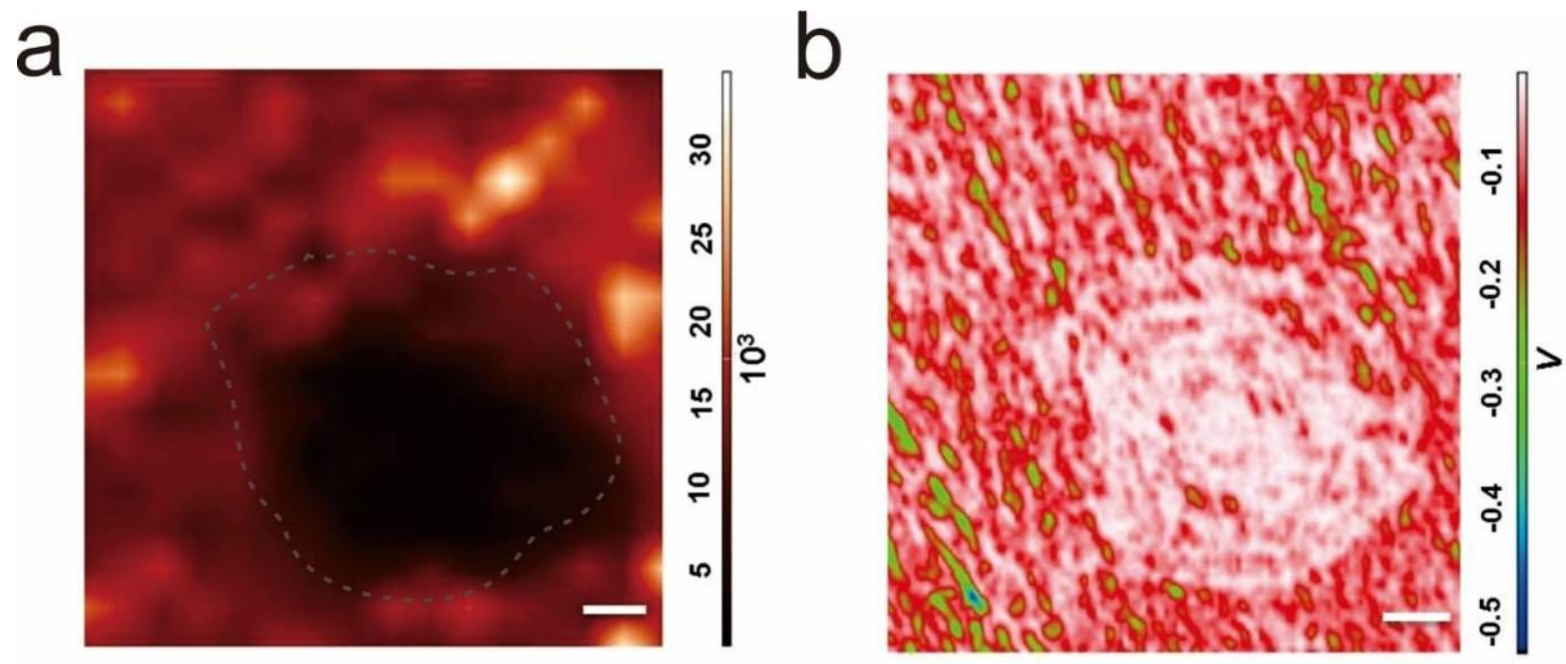

Figure S9. Raman mapping and confocal reflection microscopic (CRM) images of single PC-12 cells with a circular shape. (a) Raman mapping at $830 \mathrm{~cm}^{-1}$ and (b) confocal reflection microscopic (CRM) images of single PC-12 cells with a circular shape. The dotted line is the boundary of the PC-12 cell. The scale bar is $5 \mu \mathrm{m}$. 

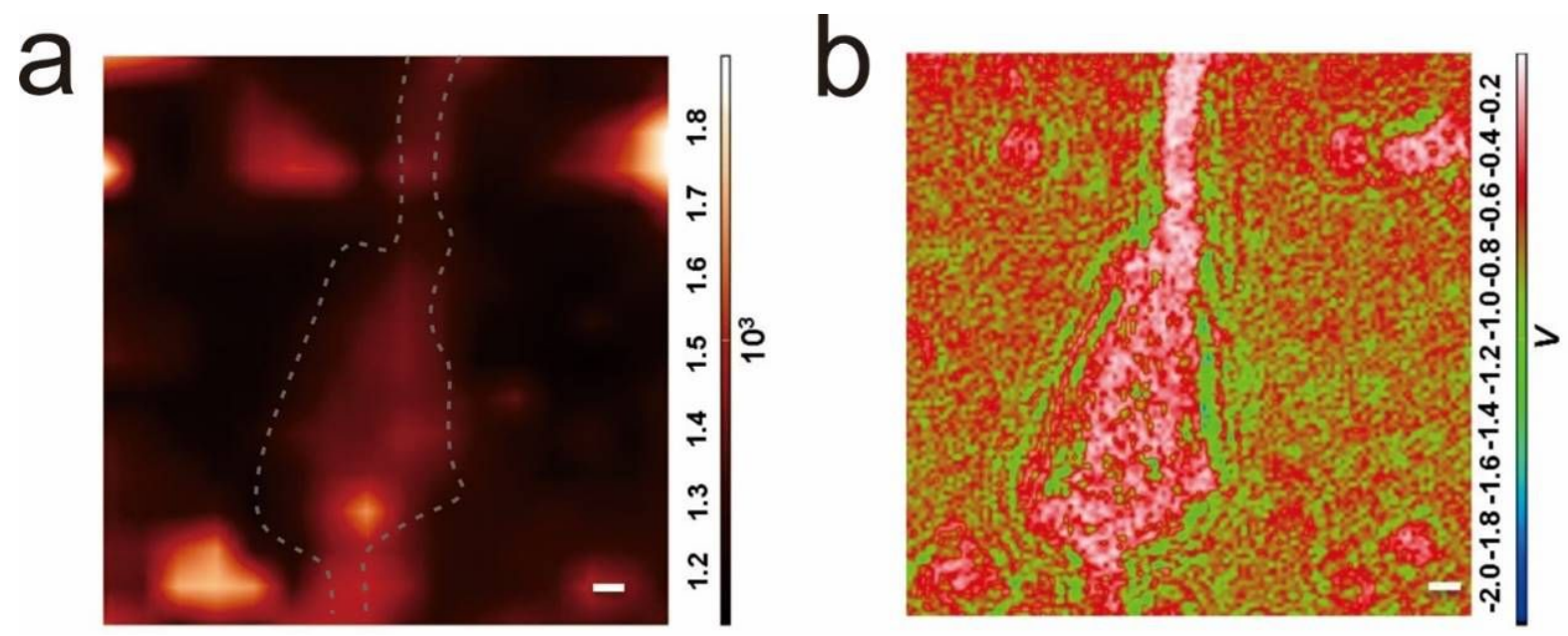

Figure S10. Raman mapping and CRM images of single PC-12 cells on hybrid nano-SERS array without MGDA aptamers. (a) Raman mapping at $830 \mathrm{~cm}^{-1}$ and (b) CRM image of single PC-12 cells on hybrid nano-SERS array without DA aptamers. The Raman intensities obtained from cell-attached areas are stronger than other areas, as opposed to the Raman mapping images of PC-12 cells on the hybrid nano-SERS array. The dotted line is the boundary of the PC-12 cell. The scale bar is $5 \mu \mathrm{m}$. 

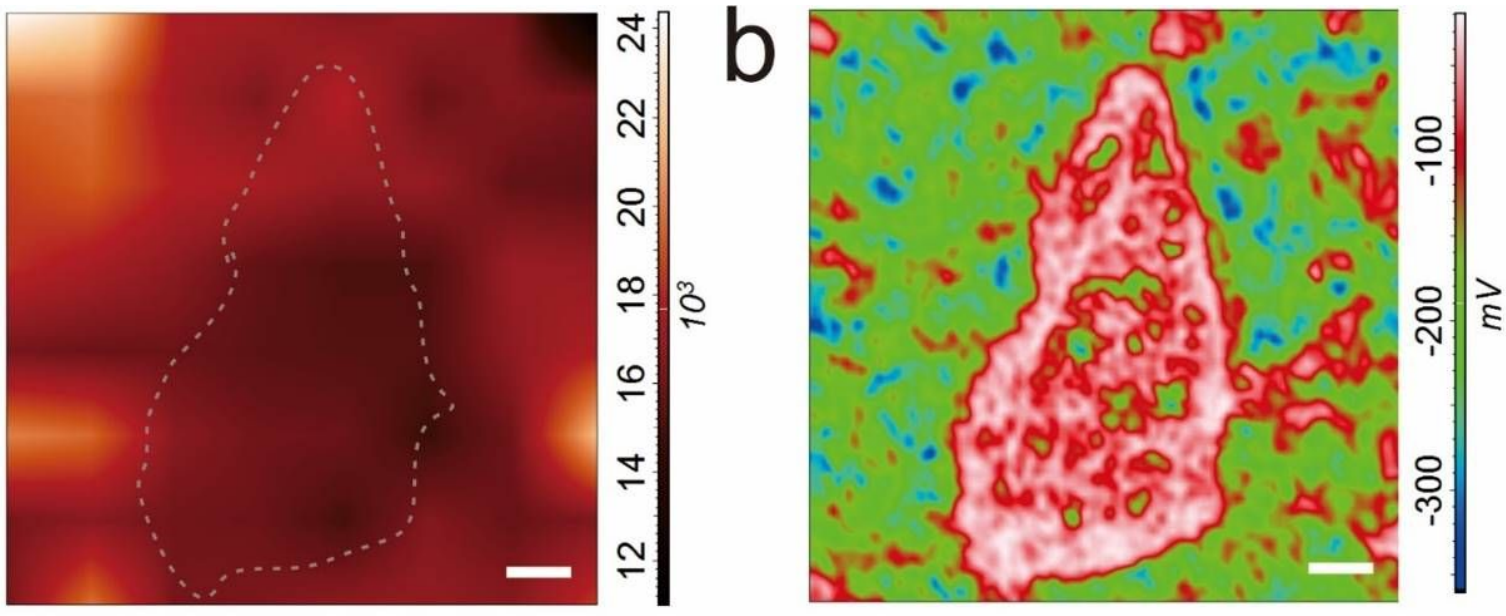

Figure S11. Raman mapping and CRM images of NE-4C cells (non-dopaminergic cells) on a hybrid nanoSERS array with MG-DA aptamers. (a) Raman mapping at $830 \mathrm{~cm}^{-1}$ and (b) CRM images of NE-4C cells on an aptamer-modified hybrid nano-SERS array. The dotted line in the (a) is the boundary of the NE-4C cell. The scale bar is $5 \mu \mathrm{m}$. 


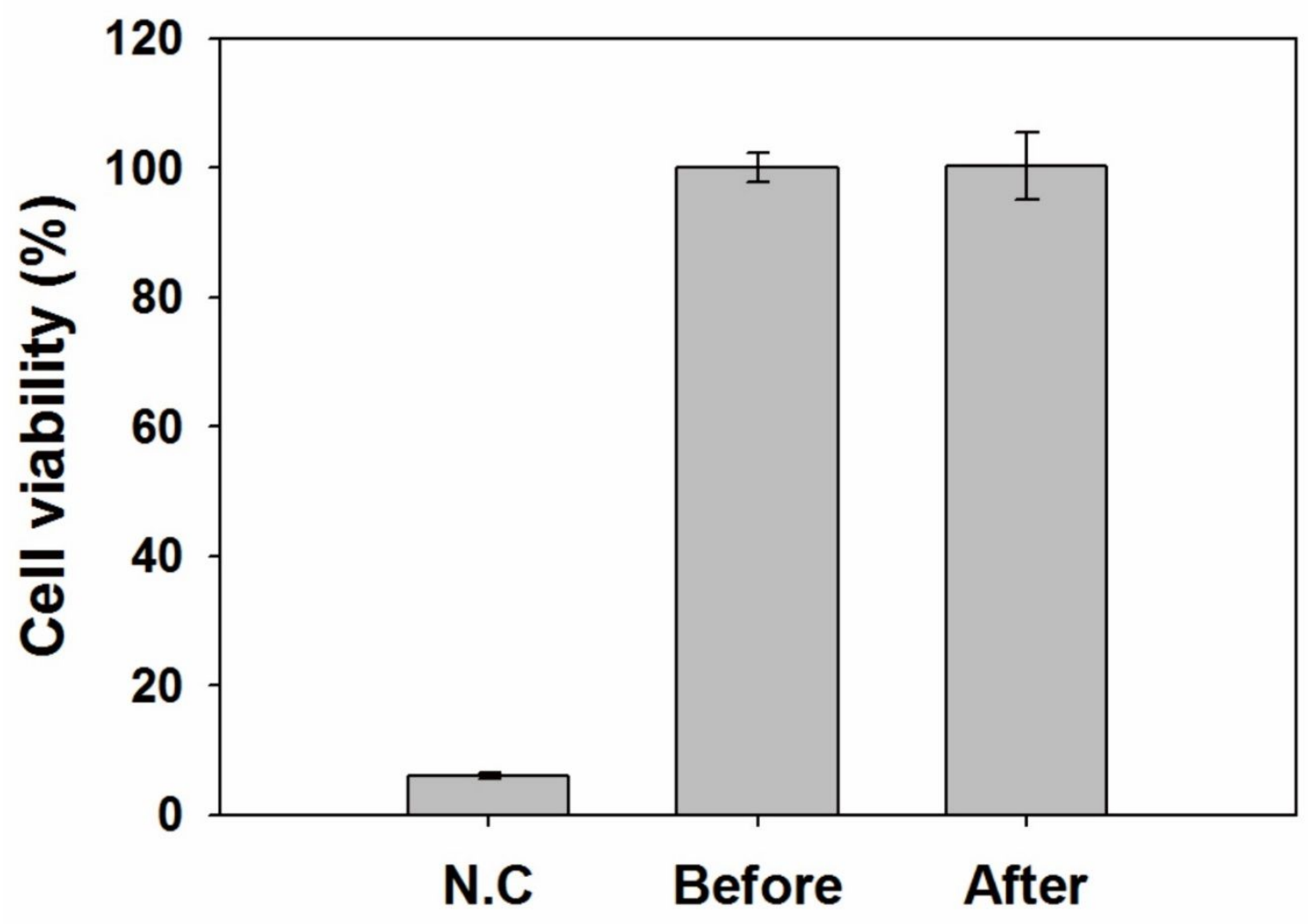

Figure S12. Cell viability test after Raman mapping of PC-12 cells on hybrid nano-SERS array. PC-12 cells before and after Raman mapping were measured by MTT (3-[4,5-dimethylthiazol-2-yl]-2,5 diphenyl tetrazolium bromide) assay. Negative control (N.C.) represented the bare nano-SERS array without any cells. 